\title{
Violation of prophylactic vancomycin administration timing is a potential risk factor for rate of surgical site infections in cardiac surgery patients: a prospective cohort study
}

\author{
Paolo Cotogni ${ }^{1 *}$, Cristina Barbero ${ }^{2}$, Roberto Passera ${ }^{3}$, Lucina Fossati ${ }^{4}$, Giorgio Olivero ${ }^{5}$ and Mauro Rinaldi ${ }^{2}$
}

\begin{abstract}
Background: Intensivists and cardiothoracic surgeons are commonly worried about surgical site infections (SSIs) due to increasing length of stay (LOS), costs and mortality. The antimicrobial prophylaxis is one of the most important tools in the prevention of SSIs. The objective of this study was to investigate the relationship between the timing of antimicrobial prophylaxis administration and the rate of SSIs.

Methods: A prospective cohort study was carried out over 1-year period in all consecutive adult patients undergoing elective cardiac surgery. The population was stratified in patients whose antimicrobial prophylaxis administration violated or not the vancomycin timing protocol (i.e., when the first skin incision was performed before the end of vancomycin infusion). To compare SSI rates, the cohort was further stratified in patients at low and high risk of developing SSIs.

Results: Over the study period, 1020 consecutive adult patients underwent cardiac surgery and according to study inclusion criteria, 741 patients were prospectively enrolled. A total of $60 \mathrm{SSIs}$ were identified for an overall infection rate of $8.1 \%$. Vancomycin prophylaxis timing protocol was violated in 305 (41\%) out of 741 enrolled patients. SSIs were observed in $3 \%$ of patients without violation of the antimicrobial prophylaxis protocol (13/436) compared with $15.4 \%$ of patients with a violation of the timing protocol $(47 / 305)(P<0.0001)$. Patients at low risk with protocol violation had a higher occurrence of SSIs $(P=0.004)$ and mortality $(P=0.03)$ versus patients at low risk without protocol violation. Similarly, patients at high risk with protocol violation had a higher occurrence of SSIs $(P<0.001)$ and mortality $(P<0.001)$ versus patients at high risk without protocol violation. The logistic regression analysis showed that internal mammary artery use $(P=0.025)$, surgical time $(P<0.001)$, intensive care unit (ICU) LOS $(P=0.002)$, high risk of developing SSIs $(P<0.001)$ and protocol violation $(P<0.001)$ were risk factors for SSI occurrence as well as age $(P=0.003)$, logistic EuroSCORE $(P<0.001)$, ICU LOS $(P<0.001)$, mechanical ventilation time $(P<0.001)$ and protocol violation $(P<0.001)$ were risk factors for mortality.
\end{abstract}

Conclusions: This study showed that violation of the timing of prophylactic vancomycin administration significantly increased the probability of SSIs and mortality from infectious cause in cardiac surgery patients.

Keywords: Antimicrobial prophylaxis, Surgical wound infection, Postoperative infectious complications

\footnotetext{
* Correspondence: paolo.cotogni@unito.it

'Department of Anesthesia and Intensive Care, S. Giovanni Battista Hospital,

University of Turin, Via Giovanni Giolitti 9, 10123 Turin, Italy

Full list of author information is available at the end of the article
} 


\section{Background}

The incidence of surgical site infections (SSIs) after cardiac surgery ranges differently according to the type of wound infection; specifically, superficial wound infection occurs in 2 to $20 \%$ of patients and deep sternal wound infection occurs in 0.25 to $5 \%$ [1-6].

Risk factors that have been linked to SSIs include features in the host such as advanced age, the presence of liver or lung dysfunction, cancer, diabetes mellitus and over- or undernutrition [7, 8]. Similarly, several operation characteristics can influence the risk of infection in cardiac surgery: skin antisepsis; length of operation; surgical technique; coronary artery bypass graft (CABG) surgery involving the use of a saphenous vein autograft that can carry bacteria from the harvest site deep into the cardiac operative site; use of the internal mammary artery (IMA) that deprives the sternum of blood supply; the use of prosthetic intracardiac or aortic implants; cardiopulmonary bypass or systemic cooling for myocardial protection; and invasive devices remaining after surgery (chest drains, pacing wires and intravenous catheters) $[1,8,9]$. Recent reports focused on an increasing number of infections caused by resistant Gram-positive pathogens, such as methicillin-resistant Staphylococcus aureus (MRSA) and coagulase-negative staphylococcus $[4,5,10]$. Compared with methicillinsensitive Staphylococcus aureus mediastinitis, MRSA mediastinitis has up to an 11-fold increased mortality rate [5].

In patients undergoing cardiac surgery, an SSI is associated with increased morbidity, prolonged length of stay and increased costs with an in-hospital mortality rate of $10-20 \%[1,6,11]$. Thus, many preventive measures were suggested as effective for reducing the incidence of SSIs, such as preoperative screening for carriage of multiresistant organisms (e.g., MRSA), antimicrobial prophylaxis, preoperative skin preparation, accurate surgical technique, postoperative glycemic control and wound management $[9,12]$. Antimicrobial intravenous prophylaxis is routinely administered to patients undergoing cardiac surgery because the benefits of preoperative antibiotic administration in these patients have been clearly demonstrated in placebo-controlled studies [13]. However, the debate over choice, dose, duration and timing of antimicrobial prophylaxis protocol is still all the rage [14].

Seminal literature demonstrated that antimicrobial prophylaxis administered too late or too early reduced the efficacy of the antibiotic and may increase the risk of SSI [7]. The definite timing of administration of the first antibiotic dose has not been assessed in randomized controlled trials; however, there is a strong rationale supporting the need for the timely administration of preoperative antimicrobial prophylaxis [7, 11, 15]. Indeed, the timing of the administration of the prophylactic antibiotic is still an important issue for the cardiac surgical community [16-19], because despite the existence of published guidelines and locally agreed protocols for the antimicrobial prophylaxis administration, often there is a gap between what is recommended and what is practiced $[15,20]$.

The primary objective of this study was to investigate the relationship between the timing of antimicrobial prophylaxis administration - with respect to surgical incision time - and the rate of SSIs, comparing cardiac surgery patients at low and high risk of infection. This objective was related to a specific exploratory mandate received from our Hospital Infection Control Committee to evaluate our policy of antimicrobial prophylaxis in cardiac surgery.

\section{Methods}

\section{Study design}

A single-centre prospective cohort study was carried out in the Department of Cardiovascular Surgery of a 1200bed tertiary care university hospital (S. Giovanni Battista Hospital). Over 1-year period, all consecutive adult patients undergoing cardiac surgery were assessed for eligibility. The exclusion criteria were renal dysfunction (on dialysis or creatinine clearance $\leq 30 \mathrm{~mL} / \mathrm{min}$, estimated by the Cockcroft-Gault formula); infectious diseases that required antibiotic therapy in the previous 2 weeks; heart and lung transplant surgery; solid or hematologic tumours as well as chemotherapy or radiation therapy in the previous 6 months; preoperative stay in intensive care unit (ICU) more than $24 \mathrm{~h}$; allergy to cefazolin or vancomycin; and emergency operations.

The study protocol was reviewed and approved by our Institutional Ethics Committee (No. 0078553) and patients provided written informed consent before their enrolment. The work was conducted in compliance with Institutional Review Board/Human Subjects Research Committee requirements.

Our protocol of antimicrobial prophylaxis was a single $1000 \mathrm{mg}$ cefazolin dose, diluted in $20 \mathrm{~mL} 0.9 \% \mathrm{NaCl}$ solution, initiated 30 to $60 \mathrm{~min}$ before surgery and administered as a slow intravenous bolus; plus a single $1000 \mathrm{mg}$ vancomycin dose, diluted in $100 \mathrm{~mL} 0.9 \% \mathrm{NaCl}$ solution, started within $2 \mathrm{~h}$ before surgery and administered over $60 \mathrm{~min}$ intravenously infusion to prevent the release of histamine. A further three doses of cefazolin $1000 \mathrm{mg}$ at 8-h intervals were given postoperatively, while no further vancomycin doses were administrated postoperatively. Since 2005, our protocol provides the choice to combine cefazolin with vancomycin for antimicrobial prophylaxis in patients undergoing cardiac surgery. The rationale for using vancomycin was an increased prevalence of MRSA infections, which exceeded $60 \%$ hospital-wide and isolates identified in cardiac 
surgery patients with SSIs. Antimicrobial prophylaxis is started in the preoperative holding area. Vancomycin protocol and timing of administration were chosen based upon recommendations of our Hospital Infection Control Committee according to the Sanford Guide [21] and the Society of Thoracic Surgeons Guidelines [16].

The study population was stratified in patients whose antimicrobial prophylaxis administration violated or not our vancomycin timing protocol. Antimicrobial prophylaxis timing protocol was considered as violated when the first surgical skin incision was performed before the end of the vancomycin infusion. A healthcare provider (i.e., physician, nurse or cardiovascular technician) was required to document the exact time the antibiotic infusion was started, as well anaesthesiologists or cardiac surgeons who recorded the exact time the first skin incision.

To compare SSI rates adequately, the cohort was further stratified in patients at low and high risk of developing SSIs according to the literature $[1,8,10]$. Specifically, patients were included in the high risk group in case of: (i) chronic liver disease (classified as Child-Pugh class B and C); (ii) insulin-dependent diabetes; (iii) body mass index (BMI) $<17$ or $>40 \mathrm{~kg} / \mathrm{m}^{2}$; (iv) steroid or other immunosuppressive drug use; (v) chronic obstructive pulmonary disease; and (vi) extracardiac arteriopathy (i.e., claudication, carotid occlusion or $>50 \%$ stenosis, amputation for arterial disease and previous or planned intervention on the abdominal aorta, limb arteries or carotids). Thus, cardiac surgery patients were assigned to four groups according to SSI risk factors and violation of the timing of antimicrobial prophylaxis protocol administration as follows: (i) low risk patients without protocol violation; (ii) low risk patients with protocol violation; (iii) high risk patients without protocol violation; and (iv) high risk patients with protocol violation.

According to Centers for Disease Control and Prevention (CDC) guidelines [8], the definition of an SSI requires that one of the following criteria be met: (i) superficial (infection above the sternum with no bone involvement); (ii) deep (infection involving the sternum and organ/space such as mediastinitis); and (iii) leg donor site infections. Patients with SSI must have positive culture results of surgical sites or drainage from the mediastinal area or evidence of infection during surgical re-exploration or fever, sternal instability and positive blood culture results [8]. Other infectious complications were defined as bloodstream infection (BSI), lower respiratory tract infection (LRTI) and urinary tract infection (UTI) according to CDC guidelines. Perioperative management and skin preparation were standardized in our Department according to CDC guidelines. According to the literature, patients were followed up for 30 days after the surgical procedure $[18,19]$. Mortality was defined as death during hospitalization or within 30 days after surgery from infectious cause.

\section{Statistical analysis}

The patients' characteristics were analysed by the Fisher's exact test for categorical variables, while by the

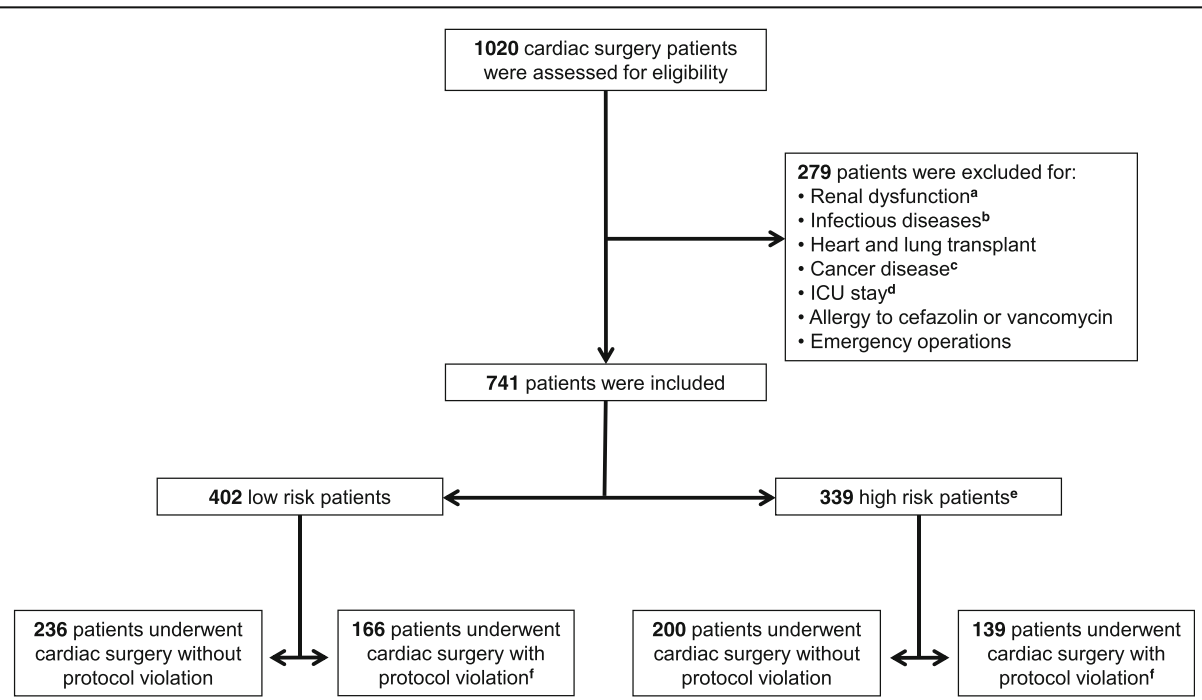

Fig. 1 Study design. ${ }^{a}$ Dialysis or creatinine clearance $\leq 30 \mathrm{~mL} / \mathrm{min}$. ${ }^{b}$ Infectious diseases that required antibiotic therapy in the previous 2 weeks. 'Patients with solid or hematologic tumours, as well as patients underwent chemotherapy or radiation therapy in the previous 6 months. dPreoperative stay in the intensive care unit (ICU) for more than $24 \mathrm{~h}$. ${ }^{e}$ Patients were considered at high risk of developing surgical site infections in case of: chronic liver disease (classified as Child-Pugh class B and C); insulin-dependent diabetes; body mass index $<17$ or $>40 \mathrm{~kg} / \mathrm{m}^{2}$; steroid or other immunosuppressive drug use; chronic obstructive pulmonary disease; and extracardiac arteriopathy (i.e., claudication, carotid occlusion or $>50 \%$ stenosis, amputation for arterial disease and previous or planned intervention on the abdominal aorta, limb arteries or carotids). ${ }^{\text {VViolation of }}$ antimicrobial prophylaxis timing protocol 
Mann-Whitney test for continuous ones; all results for the latter were expressed as the median (range). Two different independent series of univariate/multivariate binary logistic regression models were used to estimate the odds of SSI occurrence and mortality within 30 days after surgery (dependent variables), evaluating as their risk factors: gender, IMA use, high risk of developing SSIs and protocol violation (independent categorical variables) as well as age, BMI, surgical time, logistic EuroSCORE, mechanical ventilation time and ICU length of stay (LOS) (independent continuous variables). All reported P values were obtained by the two-sided exact method, at the conventional 5\% significance level. Data were analysed by R 3.3.2 (R Foundation for Statistical Computing, Vienna-A, http://www.R-project.org).

\section{Results}

Over the study period, 1020 consecutive adult patients underwent cardiac surgery. According to study inclusion criteria, 741 patients were prospectively enrolled, while 279 patients were excluded (Fig. 1). Main patients' characteristics are reported in Table 1. According to variables considered as risk factors for infectious complications, 402 patients were considered at low risk of developing SSIs and 339 were considered at high risk. Of the 741 patients included in the study, antimicrobial prophylaxis timing protocol was violated in 305 patients (41.2\%); specifically, in these patients the skin incision was performed before the end of the vancomycin infusion. No patients had vancomycin infusion more than $120 \mathrm{~min}$ prior to skin incision. No violation regarding cefazolin administration was observed.

Table 2 shows infectious complications. SSIs were 8.1\%: two-thirds were superficial wound infections of the chest, deep infections were $25 \%$ and few were at a donor site. SSIs were observed in $3 \%$ of patients without violation of the antimicrobial prophylaxis protocol (13/436) compared with $15.4 \%$ of patients with a violation of the

Table 1 Patients' characteristics

\begin{tabular}{|c|c|c|c|c|c|c|}
\hline & Low risk $(n=402)$ & & & High risk $(n=339)$ & & \\
\hline & $\begin{array}{l}\text { Without protocol } \\
\text { violation }\end{array}$ & $\begin{array}{l}\text { With protocol } \\
\text { violation }\end{array}$ & $P$ & $\begin{array}{l}\text { Without protocol } \\
\text { violation }\end{array}$ & $\begin{array}{l}\text { With protocol } \\
\text { violation }\end{array}$ & $P$ \\
\hline$n$ & 236 & 166 & & 200 & 139 & \\
\hline Age, median (range) & $70(25-86)$ & $70(34-84)$ & 0.51 & $71(37-88)$ & $71(44-88)$ & 0.50 \\
\hline Male gender, n (\%) & $149(63)$ & $100(60.2)$ & 0.63 & $124(62)$ & $74(53.2)$ & 0.13 \\
\hline $\mathrm{BMI}, \mathrm{kg} / \mathrm{m}^{2}$, median (range) & $26(18-40)$ & $26(18-39)$ & 0.81 & $28(17-43)$ & $28(17-41)$ & 0.84 \\
\hline Diabetes, n (\%) & $46(19)^{a}$ & $27(16.3)^{a}$ & 0.41 & $50(25)^{b}$ & $45(32.4)^{b}$ & 0.17 \\
\hline COPD, n (\%) & 0 & 0 & 一 & $24(12)$ & $13(9.3)$ & 0.55 \\
\hline Hypertension, n (\%) & $151(64)$ & $108(65.1)$ & 0.91 & $132(66)$ & $99(71.2)$ & 0.37 \\
\hline Smoke, n (\%) & $28(12)$ & $30(18.1)$ & 0.11 & $52(26)$ & $48(34.5)$ & 0.12 \\
\hline Surgical time, min, median (range) & $249(119-593)$ & $255(132-495)$ & 0.49 & $247(140-442)$ & $243(152-430)$ & 0.60 \\
\hline Surgical procedure, n (\%) & & & 0.08 & & & 0.11 \\
\hline CABG & $72(30.5)$ & $58(34.9)$ & & $70(35)$ & $53(38.1)$ & \\
\hline Valve & $113(47.9)$ & $59(35.5)$ & & $52(26)$ & $24(17.3)$ & \\
\hline CABG + Valve & $34(14.4)$ & $30(18.1)$ & & $24(12)$ & $12(8.6)$ & \\
\hline Other $^{\mathrm{c}}$ & $17(7.2)$ & $19(11.5)$ & & $54(27)$ & $50(36)$ & \\
\hline Off-pump CABG, n (\%) & $21(8.9)$ & $8(4.8)$ & 0.17 & $11(5.5)$ & $9(6.5)$ & 0.89 \\
\hline Left IMA, n (\%) & $53(22.4)$ & $40(24.1)$ & 0.79 & $44(22)$ & $33(23.7)$ & 0.81 \\
\hline Both IMA, n (\%) & $17(7.2)$ & $8(4.8)$ & 0.44 & $13(6.5)$ & $9(6.5)$ & $>0.99$ \\
\hline EuroSCORE additive, median (range) & $5(1-6)$ & $5(1-6)$ & 0.80 & $8(1-16)$ & $8(1-14)$ & 0.72 \\
\hline EuroSCORE logistic, median (range) & $4.8(1-7.74)$ & $4.6(1-7.21)$ & 0.41 & $9.7(1-44.45)$ & $9.9(1-61.86)$ & 0.37 \\
\hline Mechanical ventilation, $\mathrm{h}$, median (range) & $7(2-912)$ & $8(6-415)$ & 0.31 & $9(7-816)$ & $9(8-711)$ & 0.49 \\
\hline ICU stay, d, median (range) & $1(1-24)$ & $1(1-38)$ & 0.52 & $1(1-33)$ & $1(1-45)$ & 0.30 \\
\hline RBC transfusions, n, median (range) & $2(0-9)$ & $2(0-6)$ & 0.71 & $3(0-11)$ & $2(0-10)$ & 0.61 \\
\hline
\end{tabular}

$B M I$ body mass index, COPD chronic obstructive pulmonary disease, CABG coronary artery bypass grafting, IMA internal mammary artery, EuroSCORE European System for Cardiac Operative Risk Evaluation, $h$ hours, ICU intensive care unit, $d$ days, $R B C$ red blood cell

${ }^{a}$ Non-insulin-dependent diabetes

bInsulin-dependent diabetes

${ }^{c}$ Aortic, atrial or ventricular septal defect repair, and congenital surgery 
Table 2 Infectious complications

\begin{tabular}{|c|c|c|c|c|c|}
\hline & \multicolumn{2}{|l|}{ Low risk $(n=402)$} & \multicolumn{3}{|l|}{ High risk $(n=339)$} \\
\hline & Without protocol violation & With protocol violation & Without protocol violation & With protocol violation & Total \\
\hline N & 236 & 166 & 200 & 139 & 741 \\
\hline SSI, n (\%) & $3(1.3)$ & $12(7.2)^{a}$ & $10(5)^{\mathrm{b}}$ & $35(25.2)^{c, d}$ & $60(8.1)$ \\
\hline Superficial, n & 1 & 8 & 4 & 25 & $38(63.3)$ \\
\hline Deep, n & 2 & 2 & 5 & 6 & $15(25)$ \\
\hline Donor site, $\mathrm{n}$ & 0 & 2 & 1 & 4 & $7(11.7)$ \\
\hline BSI, n (\%) & $2(0.8)$ & $8(4.8)^{\mathrm{e}}$ & $12(6)^{b}$ & $49(35.2)^{c, d}$ & $71(9.6)$ \\
\hline LRTI, n (\%) & $7(3)$ & $8(4.8)$ & $25(12.5)^{f}$ & $39(23.1)^{c, d}$ & $79(10.7)$ \\
\hline UTI, n (\%) & 0 & $1(0.6)$ & $3(1.5)$ & $15(10.8)^{\mathrm{c}, \mathrm{d}}$ & $19(2.6)$ \\
\hline Mortality ${ }^{\S}, \mathrm{n}(\%)$ & $3(1.3)$ & $8(4.8)^{9}$ & $9(4.5)^{b}$ & $20(14.4)^{c, h}$ & $40(5.4)$ \\
\hline
\end{tabular}

SSI surgical site infection, BSI bloodstream infection, $L R T I$ lower respiratory trait infection, UTI urinary trait infection

${ }^{\S}$ During hospitalization or within 30 days after surgery from infectious cause

${ }^{\text {a }} P=0.004$ versus low risk group without protocol violation

${ }^{\mathrm{b}} P=0.04$ versus low risk group without protocol violation

${ }^{c} P<0.001$ versus high risk group without protocol violation

${ }^{\mathrm{d}} P<0.001$ versus low risk group with protocol violation

${ }^{\mathrm{e}} P=0.01$ versus low risk group without protocol violation

${ }^{f} P<0.001$ versus low risk group without protocol violation

${ }^{9} P=0.03$ versus low risk group without protocol violation

${ }^{h} P=0.003$ versus low risk group with protocol violation

timing protocol $(47 / 305)(P<0.0001)$. Patients at low risk with protocol violation had a higher occurrence of SSIs $(P=0.004)$, BSIs $(P=0.01)$ and mortality $(P=0.03)$ versus patients at low risk without protocol violation. Patients at high risk with protocol violation had a higher occurrence of SSIs $(P<0.001)$, BSIs $(P<0.001)$, LRTIs $(P<0.001)$, UTIs $(P<0.001)$ and mortality $(P<0.001)$ versus patients at high risk without protocol violation. Patients at high risk without violation of the antimicrobial prophylaxis protocol had a higher occurrence of SSIs $(P=0.04)$, BSIs $(P=0.04)$, LRTIs $(P<0.001)$ and mortality $(P=0.04)$ versus patients at low risk without protocol violation. Patients at high risk with protocol violation had a higher occurrence of SSIs $(P<0.001)$, BSIs $(P<0.001)$, LRTIs $(P<0.001)$, UTIs $(P<0.001)$ and mortality $(P=0.003)$ versus patients at low risk with protocol violation.

The logistic regression analysis showed that IMA use $(P=0.025)$, surgical time $(P<0.001)$, ICU LOS $(P=0.002)$, high risk of developing SSIs $(P<0.001)$ and protocol violation $(P<0.001)$ were risk factors for SSI occurrence (Table 3$)$ as well as age $(P=0.003)$, logistic EuroSCORE $(P<0.001)$, ICU LOS $(P<0.001)$, mechanical ventilation time $(P<0.001)$ and protocol violation $(P<0.001)$ were risk factors for mortality (Table 4$)$.

Ninety-two pathogens isolated in 60 SSIs are shown in Table 5. Specifically, Gram-positive, Gram-negative and fungi were isolated in 48,40 and $12 \%$, respectively. Pathogens isolated in SSIs by groups are depicted in Table 6 . Methicillin-resistant Staphylococci (aureus, coagulase-

Table 3 Risk factors for surgical site infections (SSIs)

\begin{tabular}{|c|c|c|c|c|c|c|}
\hline & \multicolumn{3}{|c|}{$\begin{array}{l}\text { Logistic regression } \\
\text { Univariate models }\end{array}$} & \multicolumn{3}{|c|}{$\begin{array}{l}\text { Logistic regression } \\
\text { Multivariate model }\end{array}$} \\
\hline & Odds ratio & $95 \% \mathrm{Cl}$ & $P$ & Odds ratio & $95 \% \mathrm{Cl}$ & $P$ \\
\hline Body mass index & 1.07 & $1.02-1.13$ & 0.007 & 1.01 & $0.94-1.08$ & 0.781 \\
\hline Internal mammary artery use & 1.85 & $1.08-3.17$ & 0.026 & 2.11 & $1.10-4.04$ & 0.025 \\
\hline Surgical time & 1.01 & $1.01-1.02$ & $<0.001$ & 1.01 & $1.01-1.02$ & $<0.001$ \\
\hline Intensive care unit LOS & 1.12 & $1.09-1.16$ & $<0.001$ & 1.06 & $1.02-1.10$ & 0.002 \\
\hline High risk ${ }^{a}$ & 3.95 & $2.16-7.22$ & $<0.001$ & 4.70 & $2.32-9.53$ & $<0.001$ \\
\hline Protocol violation ${ }^{\mathrm{b}}$ & 5.93 & $3.15-11.17$ & $<0.001$ & 7.03 & $3.41-14.52$ & $<0.001$ \\
\hline
\end{tabular}

Cl Confidence interval, LOS length of stay

a High risk of developing SSIs according to the literature $[1,8,10]$; in case of: (i) chronic liver disease (classified as Child-Pugh class B and C); (ii) insulin-dependent diabetes; (iii) body mass index $<17$ or $>40 \mathrm{~kg} / \mathrm{m} 2$; (iv) steroid or other immunosuppressive drug use; (v) chronic obstructive pulmonary disease; and (vi) extracardiac arteriopathy (i.e.,claudication, carotid occlusion or $>50 \%$ stenosis, amputation for arterial disease and previous or planned intervention on the abdominal aorta, limb arteries or carotids)

${ }^{\mathrm{b}}$ Antimicrobial prophylaxis timing protocol was considered as violated when the first surgical skin incision was performed before the end of the vancomycin infusion 
Table 4 Risk factors for mortality ${ }^{\mathrm{a}}$

\begin{tabular}{|c|c|c|c|c|c|c|}
\hline & \multicolumn{3}{|c|}{$\begin{array}{l}\text { Logistic regression } \\
\text { Univariate models }\end{array}$} & \multicolumn{3}{|c|}{$\begin{array}{l}\text { Logistic regression } \\
\text { Multivariate model }\end{array}$} \\
\hline & Odds ratio & $95 \% \mathrm{Cl}$ & $P$ & Odds ratio & $95 \% \mathrm{Cl}$ & $P$ \\
\hline Age & 1.13 & $1.07-1.19$ & $<0.001$ & 1.15 & $1.05-1.26$ & 0.003 \\
\hline EuroSCORE logistic & 1.24 & $1.17-1.31$ & $<0.001$ & 1.21 & $1.11-1.33$ & $<0.001$ \\
\hline Intensive care unit LOS & 1.25 & $1.20-1.31$ & $<0.001$ & 1.14 & $1.07-1.21$ & $<0.001$ \\
\hline Mechanical ventilation time & 1.34 & $1.24-1.45$ & $<0.001$ & 1.18 & $1.08-1.29$ & $<0.001$ \\
\hline Protocol violation ${ }^{\mathrm{b}}$ & 3.57 & $1.79-7.14$ & $<0.001$ & 10.16 & $2.48-41.58$ & $<0.001$ \\
\hline
\end{tabular}

Cl Confidence interval, LOS length of stay

aDuring hospitalization or within 30 days after surgery from infectious cause

${ }^{\mathrm{b}}$ Antimicrobial prophylaxis timing protocol was considered as violated when the first surgical skin incision was performed before the end of the vancomycin infusion

negative and hominis) accounted for $28 \%$ of pathogens. When we studied methicillin-resistance or vancomycin susceptibility of Gram-positive isolates according to the timing of antimicrobial prophylaxis none of the differences between groups in the rate of such resistances reached statistical significance. No clusters of any specific pathogen were noted during the study period.

\section{Discussion}

In this study, cardiac surgery population was divided in patients at high risk of developing infections because of well-known risk factors and patients at low risk. As expected, patients at high risk (i.e., patients with severe comorbidities, immunosuppressive therapy, severe obesity or malnutrition) had a significant higher occurrence of SSIs as well as of BSIs, LRTIs and mortality compared with patients at low risk, independently of violation of the antimicrobial prophylaxis protocol. This finding reflects a population of patients who were more severely ill and therefore at higher risk for postoperative infectious complications.

The Society of Thoracic Surgeons Practice Guidelines on antibiotic prophylaxis in cardiac surgery recommended that in the setting of the institutional presence of a 'high incidence' of MRSA, it would be reasonable to combine a $\beta$-lactam (cefazolin) with a glycopeptide (vancomycin) for prophylaxis (Class IIB recommendation, Level of Evidence C) [16]. Optimal dosage regimens

Table 5 Pathogens isolated in 60 surgical site infections

\begin{tabular}{ll}
\hline Pathogens, n & 92 \\
Gram-positive organisms, n (\%) & $44(48)$ \\
Staphylococcus aureus, n & 23 \\
Coagulase-negative staphylococci, n & 11 \\
Enterococcus spp, n & 9 \\
Streptococci, n & 1 \\
Gram-negative organisms, n (\%) & $37(40)$ \\
Fungi, n (\%) & $11(12)$ \\
\hline
\end{tabular}

of vancomycin and protocol of administration still remain controversial [16, 22]. The Society of Thoracic Surgeons Guidelines mentioned that any of the following doses and durations may be used: $1000 \mathrm{mg}, 1500 \mathrm{mg}$, or $15 \mathrm{mg} / \mathrm{kg}$; and $24 \mathrm{~h}$ versus $48 \mathrm{~h}$ or 1 dose versus 2 doses $[16,22]$. Specifically, guidelines for appropriate dosing of prophylactic antibiotics stated that 'In patients for whom vancomycin is an appropriate prophylactic antibiotic for cardiac surgery, a dose of 1 to $1.5 \mathrm{~g}$ or a weight-adjusted dose of $15 \mathrm{mg} / \mathrm{kg}$ administered intravenously slowly over $1 \mathrm{~h}$, with completion within $1 \mathrm{~h}$ of the skin incision, is recommended' (Class I, Level of Evidence A) [16]. Similarly, the 2011 American College of Cardiology/American Heart Association guideline for CABG surgery recommended that Antibiotic prophylaxis should be initiated 30 to $60 \mathrm{~min}$ before surgery, usually at the time of anaesthetic induction, except for vancomycin, which should be started $2 \mathrm{~h}$ before surgery and infused slowly' [6]. Finally, the Scottish Intercollegiate Guidelines Network, updated April 2014, stated that 'Vancomycin should be given by intravenous infusion starting $90 \mathrm{~min}$ prior to skin incision' (Class B recommendation) [9].

Several studies investigated the association between measure(s) applied for reducing the rate of SSIs and their occurrence [23, 24]. Our study focused on the timing of antimicrobial prophylaxis; specifically, on the relationship between the first skin incision and the end of vancomycin infusion. We found that the initial surgical incision was performed before the vancomycin infusion had been completed in nearly $40 \%$ of patients. Generally, the reason for the violation of the antimicrobial prophylaxis protocol was due to our policy to start antimicrobial prophylaxis in the preoperative holding area under supervision of anaesthesiologists. This policy was adopted in our Cardiovascular Surgery Unit following the occurrence of some relevant adverse drug reactions due to vancomycin administration (i.e., mainly hypotension; occasionally, red man syndrome) [23, 24].

Differently, in the Garey's study [25] cardiac surgery patients were assigned to five groups on the basis of the 
Table 6 Pathogens isolated in surgical site infections by groups

\begin{tabular}{|c|c|c|c|c|c|}
\hline & \multicolumn{2}{|l|}{ Low risk $(n=402)$} & \multicolumn{2}{|l|}{ High risk $(n=339)$} & \multirow[t]{2}{*}{ Total } \\
\hline & Without protocol violation & With protocol violation & Without protocol violation & With protocol violation & \\
\hline SSI, n (\%) & 3/236 (1.3) & $12 / 166(7.2)$ & $10 / 200(5)$ & $35 / 139(25.2)$ & $60 / 741(8.1)$ \\
\hline Pathogen, $\mathrm{n}$ & 3 & 24 & 12 & 53 & 92 \\
\hline Gram-positive, n (\%) & $1(33)$ & $11(46)$ & $5(42)$ & $27(51)$ & $44(48)$ \\
\hline Methicillin-sensitive, $n$ & 1 & 3 & 2 & 12 & 18 \\
\hline Methicillin-resistant, n & 0 & 8 & 3 & 15 & 26 \\
\hline \multicolumn{6}{|l|}{ Vancomycin susceptibility } \\
\hline $\mathrm{MIC} \leq 1, \mathrm{n}$ & 1 & 9 & 3 & 17 & 30 \\
\hline $\mathrm{MIC}=2, \mathrm{n}$ & 0 & 2 & 1 & 9 & 12 \\
\hline$M I C \geq 4, n$ & 0 & 0 & 1 & 1 & 2 \\
\hline Gram-negative, n (\%) & $1(33)$ & $12(50)$ & $4(33)$ & $20(38)$ & $37(40)$ \\
\hline Fungi, n (\%) & $1(33)$ & $1(4)$ & $3(25)$ & $6(11)$ & $11(12)$ \\
\hline
\end{tabular}

Multiple pathogens were identified in some patients; therefore, total pathogens identified do not add up to the total number of SSIs. SSI Surgical Site Infection, MIC minimum inhibitory concentration

relation between the start time of the vancomycin infusion and the time of the initial surgical incision. In this study, antibiotic prophylaxis was started in the preoperative holding area only for the first surgical case of the day and in admission unit for all subsequent cases immediately prior to transferring the patient to the preoperative holding area. These Authors reported that of the 2048 patients in the study, $0.7 \%$ received vancomycin $0-15$ min before incision, $8.6 \%$ 16-60 $\mathrm{min}$ before incision, 43.4\% 61120 min before incision, $34.2 \%$ 121-180 min before incision and $13.1 \%>180 \mathrm{~min}$ before incision.

The relationship between the timing of antimicrobial prophylaxis and the occurrence of SSIs has been studied with conflicting results. The Surgical Care Improvement Project measure assesses compliance for antimicrobial prophylaxis administration initiated within $60 \mathrm{~min}$ (or $120 \mathrm{~min}$ for vancomycin) prior to surgical incision [11]. The choice of the preincision 60-min window for antimicrobial prophylaxis was based on two types of evidence: pharmacokinetics of the antibiotics and one large cohort study analyzing the association between timing of antibiotic administration and SSIs in several types of surgical procedures [11].

However, following studies investigating this relationship did not clearly demonstrate the superiority of the 60-min window [17-20]; in particular, some studies showed lower risk of SSI with shorter times between antibiotic administration and skin incision. Garey et al. reported that SSI developed in $26.7 \%$ of cardiac surgery patients who received vancomycin $0-15 \mathrm{~min}$ before incision, $3.4 \%$ of patients between 16 and $60 \mathrm{~min}$ before incision, $7.7 \%$ of patients between 61 and $120 \mathrm{~min}$ before incision, 6.9\% of patients between 121 and $180 \mathrm{~min}$ before incision and 7.8\% of patients $>180 \mathrm{~min}$ before incision [25]. Steinberg et al. in an observational study (43.6\% were cardiac patients) found a trend toward lower risk of SSI occurring when antimicrobial prophylaxis with vancomycin or cephalosporins were given within 60 and 30 min prior to incision, respectively [18]. Hawn et al. in a retrospective study in noncardiac surgery patients found that the SSI risk was not significantly associated with prophylactic antibiotic timing [19].

SSIs are still among the most severe complications in cardiac surgery patients. The overall SSI rate observed in our study was $8.1 \%$, which was similar or lower to that previously reported [1-3]. The main finding of our study was that violation of the timing of vancomycin prophylaxis protocol was a significant risk factor for development of SSI in patients undergoing cardiac surgery. Specifically, when the first surgical skin incision was performed before the end of the vancomycin infusion, we observed a 5-fold increased rate of SSIs both in low and high risk patients.

Nosocomial infections occur in 10 to $20 \%$ of cardiac surgery patients [6]; however, while SSIs incur significant morbidity and costs but rarely lead to death, conversely, postoperative LRTI, BSI and endocarditis are more frequently correlated with mortality [15]. In our study, we also found a consistent relationship between violation of vancomycin prophylaxis timing protocol and rates of postoperative infectious complications as well as mortality from infectious cause. Specifically, BSIs and mortality were increased 6-fold and more than 3-fold, respectively both in low and high risk patients. Moreover, LRTIs and UTIs were increased 2-fold and 7-fold, respectively in high risk patients. Also for mortality, violation of the timing of vancomycin prophylaxis protocol was a significant risk factor.

Actually, before starting this study we did not suspect that the timing of vancomycin prophylaxis administration was being violated at this rate as well as that this violation 
was associated with a significantly increased rate of SSIs, postoperative infectious complications and mortality from infectious cause. However, the overall SSI rate and mortality observed in our study were similar or lower to those previously reported in other studies in cardiac surgery patients [1-6].

Policies and practices aimed at reducing the risk of SSIs include performing surveillance for SSIs as well as measuring and providing feedback to healthcare providers on the rates of compliance with process measures, including antimicrobial prophylaxis [7, 12]. This study let us to discover that the violation of the protocol was due to the start of antimicrobial prophylaxis in the preoperative holding area. Indeed, the information obtained in this study was reported to our healthcare providers and has altered practice patterns for avoiding the persistent risk of violation of prophylactic vancomycin administration timing.

\section{Strengths and limitations of the study}

If compared with previous studies, our study has some relevant features: (1) it was a prospective study; (2) data were collected through a clinical study and not from a database or registry; (3) antibiotic timing data were collected in 'real-time' in the operating room and not from the patient chart; (4) only cardiac surgery patients were enrolled; (5) all patients received the same prophylactic administration of antibiotics; (6) the rate of BSIs, LRTIs, UTIs and mortality from infectious cause were also investigated; (7) it was concluded in only 12 months and (8) no patient was lost to follow-up. Moreover, to the best of our knowledge, this is the first study investigating the relationship between the rate of SSIs in cardiac surgery patients and the presence or absence of violation of the timing of antimicrobial prophylaxis administration, comparing patients at low and high risk of infections.

The study presented several limitations. First, it was a single-centre study. Second, neither a calculation was made on the number of subjects required nor an interim analysis was conducted since the study was designed by our statistician to be continuous over one year. Specifically, the duration of a year was necessary to enrol an adequate number of patients (i.e., 741) to obtain statistically significant differences among the groups. As a matter of fact, the earlier studies enrolled a number of patients ranging among 2048 and 4472 [18, 25].

Moreover, being an observational study, there was no randomization of patients to the two groups (with and without protocol violation), although the characteristics of patients in the two groups turned out not to be statistically different. Finally, in the study period patients were not screened for S. aureus colonization prior to surgery.

\section{Conclusions}

The variability in antimicrobial prophylaxis timing significance among results reported in the literature suggests that the association between timing and SSIs is greatly related to the surgical population, the antibiotic(s) and the timing intervals investigated.

Despite some limitations, our study showed that violation of the timing of prophylactic vancomycin administration significantly increased the probability of SSIs and mortality from infectious cause in patients undergoing cardiac surgery.

\begin{abstract}
Abbreviations
BMI: Body mass index; BSI: Bloodstream infection; CABG: Coronary artery bypass graft surgery; CDC: Centers for Disease Control and Prevention; EuroSCORE: European system for cardiac operative risk evaluation; ICU: Intensive care unit; IMA: Internal mammary artery; LOS: Length of stay; LRTI: Lower respiratory tract infection; MRSA: Methicillin-resistant

Staphylococcus aureus; SSI: Surgical site infection; UTI: Urinary tract infection
\end{abstract}

\section{Acknowledgements}

We thank all anaesthesiologists, cardiac surgeons, nurses and cardiovascular perfusionists of the Cardiovascular Surgery for their continuous support during the study.

Funding

This work was partially supported by the Regione Piemonte (Italy) (grant No. 2472/DA2001 to PC).

\section{Availability of data and materials}

The dataset of this study is deposited in the repositories of Regional Public Healthcare Office (Regione Piemonte) according to our Institutional Review Board/Human Subjects Research Committee (Comitato Etico Interaziendale A.O.U. San Giovanni Battista di Torino - A.O. C.T.O. Maria Adelaide di Torino) requirements for research projects supported by the Regione Piemonte (Italy) and is available on request (Sig.ra Di Sipio).

\section{Authors' contributions}

PC designed the study and drafted the manuscript. CB participated in the study design, carried out the study and helped to draft the manuscript. RP participated in the study design and performed the statistical analysis. LF participated in the study design and performed the microbiological analysis. GO participated in the study design and revised it critically for important intellectual content. MR participated in the study design and coordination and revised it critically for important intellectual content. All authors read and approved the final manuscript.

\section{Competing interests}

The authors declare that they have no competing interests.

\section{Consent for publication \\ Not applicable.}

\section{Ethics approval and consent to participate}

The study protocol was reviewed and approved by our Institutional Ethics Committee (Comitato Etico Interaziendale A.O.U. San Giovanni Battista di Torino - A.O. C.T.O. Maria Adelaide di Torino);(No. 0078553) and patients provided written informed consent before their enrolment. The consent to participate was obtained from cardiac surgeons. The work was conducted in compliance with Institutional Review Board/Human Subjects Research Committee requirements.

\section{Publisher's Note}

Springer Nature remains neutral with regard to jurisdictional claims in published maps and institutional affiliations. 


\section{Author details}

'Department of Anesthesia and Intensive Care, S. Giovanni Battista Hospital, University of Turin, Via Giovanni Giolitti 9, 10123 Turin, Italy. ${ }^{2}$ Department of Cardiovascular Surgery, S. Giovanni Battista Hospital, University of Turin, Turin, Italy. ${ }^{3}$ Nuclear Medicine Unit, S. Giovanni Battista Hospital, University of Turin, Turin, Italy. ${ }^{4}$ Microbiology and Virology Laboratory, S. Giovanni Battista Hospital, University of Turin, Turin, Italy. ${ }^{5}$ Department of Surgical Sciences, S. Giovanni Battista Hospital, University of Turin, Turin, Italy.

Received: 8 January 2016 Accepted: 1 March 2017

Published online: 08 March 2017

\section{References}

1. Ridderstolpe $L$, Gill H, Granfeldt $H$, Ahlfeldt $H$, Rutberg $H$. Superficial and deep sternal wound complications: incidence, risk factors and mortality. Eur J Cardiothorac Surg. 2001;20:1168-75.

2. Salehi Omran A, Karimi A, Ahmadi SH, Davoodi S, Marzban M, Movahedi N et al. Superficial and deep sternal wound infection after more than 9000 coronary artery bypass graft (CABG): incidence, risk factors and mortality. BMC Infect Dis. 2007:7:112.

3. Filsoufi F, Castillo JG, Rahmanian PB, Broumand SR, Silvay G, Carpentier A, et al. Epidemiology of deep sternal wound infection in cardiac surgery. J Cardiothorac Vasc Anesth. 2009;23:488-94.

4. Kanafani ZA, Arduino JM, Muhlbaier LH, Kaye KS, Allen KB, Carmeli Y, et al. Incidence of and preoperative risk factors for Staphylococcus aureus bacteremia and chest wound infection after cardiac surgery. Infect Control Hosp Epidemiol. 2009;30:242-8

5. Tom TS, Kruse MW, Reichman RT. Update: Methicillin-resistant Staphylococcus aureus screening and decolonization in cardiac surgery. Ann Thorac Surg. 2009;88:695-702.

6. Hillis LD, JL SPKa, Bittl JA, Bridges CR, Byrne JG, et al. 2011 ACCF/AHA guideline for coronary artery bypass graft surgery. A report of the American College of Cardiology Foundation/American Heart Association Task Force on Practice Guidelines. Developed in collaboration with the American Association for Thoracic Surgery, Society of Cardiovascular Anesthesiologists and Society of Thoracic Surgeons. J Am Coll Cardiol. 2011;58:123-210.

7. Classen DC, Evans RS, Pestotnik SL, Horn SD, Menlove RL, Burke JP. The timing of prophylactic administration of antibiotics and the risk of surgicalwound infection. N Engl J Med. 1992;326:281-6.

8. Mangram AJ, Horan TC, Pearson ML, Silver LC, Jarvis WR. Guideline for prevention of surgical site infection, 1999. Centers for disease control and prevention (CDC) hospital infection control practices advisory committee. Am J Infect Control. 1999;27:97-132.

9. Scottish Intercollegiate Guidelines Network. Antibiotic Prophylaxis in Surgery: A National Clinical Guideline. July 2008, updated April 2014. http:// www.sign.ac.uk/pdf/sign104.pdf. Accessed 1 Dec 2015.

10. Harbarth S, Huttner B, Gervaz P, Fankhauser C, Chraiti MN, Schrenzel J, et al. Risk factors for methicillin-resistant Staphylococcus aureus surgical site infection. Infect Control Hosp Epidemiol. 2008;29:890-3.

11. Bratzler DW, Hunt DR. The surgical infection prevention and surgical care improvement projects: national initiatives to improve outcomes for patients having surgery. Clin Infect Dis. 2006;43:322-30.

12. Yokoe DS, Mermel LA, Anderson DJ, Arias KM, Burstin H, Calfee DP, et al. A compendium of strategies to prevent healthcare-associated infections in acute care hospitals. Infect Control Hosp Epidemiol. 2008:29:S12-21.

13. Kreter B, Woods M. Antibiotic prophylaxis for cardiothoracic operations. Meta-analysis of thirty years of clinical trials. J Thorac Cardiovasc Surg. 1992;104:590-9.

14. Cotogni P, Barbero C, Rinaldi M. Deep sternal wound infection after cardiac surgery: evidences and controversies. World J Crit Care Med. 2015;4:265-73.

15. Lador A, Nasir H, Mansur N, Sharoni E, Biderman P, Leibovici L, et al. Antibiotic prophylaxis in cardiac surgery: systematic review and metaanalysis. J Antimicrob Chemother. 2012;67:541-50.

16. Engelman RM, Shahian D, Shemin R, Guy TS, Bratzler D, Edwards F, et al. The Society of Thoracic Surgeons practice guideline series: antibiotic prophylaxis in cardiac surgery, part II: antibiotic choice. Ann Thorac Surg. 2007:83:1569-76

17. Weber WP, Marti WR, Zwahlen M, Misteli H, Rosenthal R, Reck S, et al. The timing of surgical antimicrobial prophylaxis. Ann Surg. 2008;247:918-26.

18. Steinberg JP, Braun BI, Hellinger WC, KuseK L, Bozikis MR, Bush AJ, et al. Timing of antimicrobial prophylaxis and the risk of surgical site infections: results from the trial to reduce antimicrobial prophylaxis errors. Ann Surg. 2009;250:10-6.

19. Hawn MT, Richman JS, Vick CC, Deierhoi RJ, Graham LA, Henderson WG, et al. Timing of surgical antibiotic prophylaxis and the risk of surgical site infection. JAMA Surg. 2013;148:649-57.

20. Miliani K, L'Heriteau F, Astagneau P, INCISO Network Study Group. Noncompliance with recommendations for the practice of antibiotic prophylaxis and risk of surgical site infection: results of a multilevel analysis from the INCISO Surveillance Network. J Antimicrob Chemother. 2009;64:1307-15.

21. Gillbert DN, Moellering RC, Eliopoulos GM, Sande MA. The Sanford guide to antimicrobial therapy. 37th ed. Sperryville: Antimicrobial Therapy, Inc; 2007. p. 160

22. Edwards FH, Engelman RM, Houck P, Shahian DM, Bridges CR, Society of Thoracic Surgeons. The Society of Thoracic Surgeons practice guideline series: antibiotic prophylaxis in cardiac surgery, part I: duration. Ann Thorac Surg. 2006:81:397-404

23. Cotogni P, Passera R, Barbero C, Gariboldi A, Moscato D, Izzo G, et al. Intraoperative vancomycin pharmacokinetics in cardiac surgery with or without cardiopulmonary bypass. Ann Pharmacother. 2013;47:455-63.

24. Kritchevsky SB, Braun BI, Bush AJ, Bozikis MR, Kusel L, Burke JP, et al. The effect of a quality improvement collaborative to improve antimicrobial prophylaxis in surgical patients: a randomized trial. Ann Intern Med. 2008; 149:472-80.

25. Garey KW, Dao T, Chen H, Amrutkar P, Kumar N, Reiter M, et al. Timing of vancomycin prophylaxis for cardiac surgery patients and the risk of surgical site infections. J Antimicrob Chemother. 2006;58:645-50.

\section{Submit your next manuscript to BioMed Central and we will help you at every step:}

- We accept pre-submission inquiries

- Our selector tool helps you to find the most relevant journal

- We provide round the clock customer support

- Convenient online submission

- Thorough peer review

- Inclusion in PubMed and all major indexing services

- Maximum visibility for your research

Submit your manuscript at www.biomedcentral.com/submit
) Biomed Central 